\title{
SPECTROSCOPIC STUDIES OF 89 HER AND HD 161796
}

\author{
Sunetra Giridhar \\ Indian Institute of Astrophysics \\ Bangalore 560034, India \\ A.A.Ferro and L.E.Parrao \\ National University of Mexico \\ Dept. of Astronomy, Mexico City
}

ABSTRACT. We have studied the spectra of UU Her stars 89 Her and HD 161796 in the red spectral region. The atmospheric parameters are derived using model atmospheres and spectrum synthesis. LiI line at $6707.8 A^{\circ}$ is identified and measured in 89 Her leading to $\log (\mathrm{Li} / \mathrm{H})=2.3$. The variation of the $H \alpha$ line profile for these stars is also discussed.

INTRODUCTION

89 Her and HD 161796 are members of a group of semi-regular variables that are seen at large galactic latitudes. General behaviour of these stars is summarized by Sasselov (1984). Infrared excess has been reported for these two stars by Parthasarathy and Pottasch (1986). We felt the need for detailed spectroscopic investigation of these objects; our preliminary results are reported here.

\section{OBSERVATIONS}

The high resolution spectra covering a spectral range $5600-7000 A^{\circ}$ were taken by one of US (AAF) at DDO using $1.88 \mathrm{~m}$ reflector during 1981. Spectroscopic reductions were done at IIA, Bangalore, using RESPECT sof tware (cf Prabhu et al. 1987).

RESULTS AND DISCUSSION

The spectra of these objects resemble those of $F$ type supergiants with strong FeII and SiII lines. The most interesting feature in the spectrum of $89 \mathrm{Her}$ is LiI line at $6707.8 \mathrm{~A}^{\circ}$. Figure 1 shows the spectral region around $L i I$ line. Figures 2 and 3 show $H \alpha$ line profile variation for HD 161796 and 89 Her. P Cygni nature of the profile is obvious in the case of $89 \mathrm{Her}$ with emission component seen in longer wavelength wing of the line suggesting a scattering envelope. Also the deep absorption core indicating a large optical depth might have been caused by extended atmosphere or circumstellar shell. HD161796 does not display emission component but a sharp 

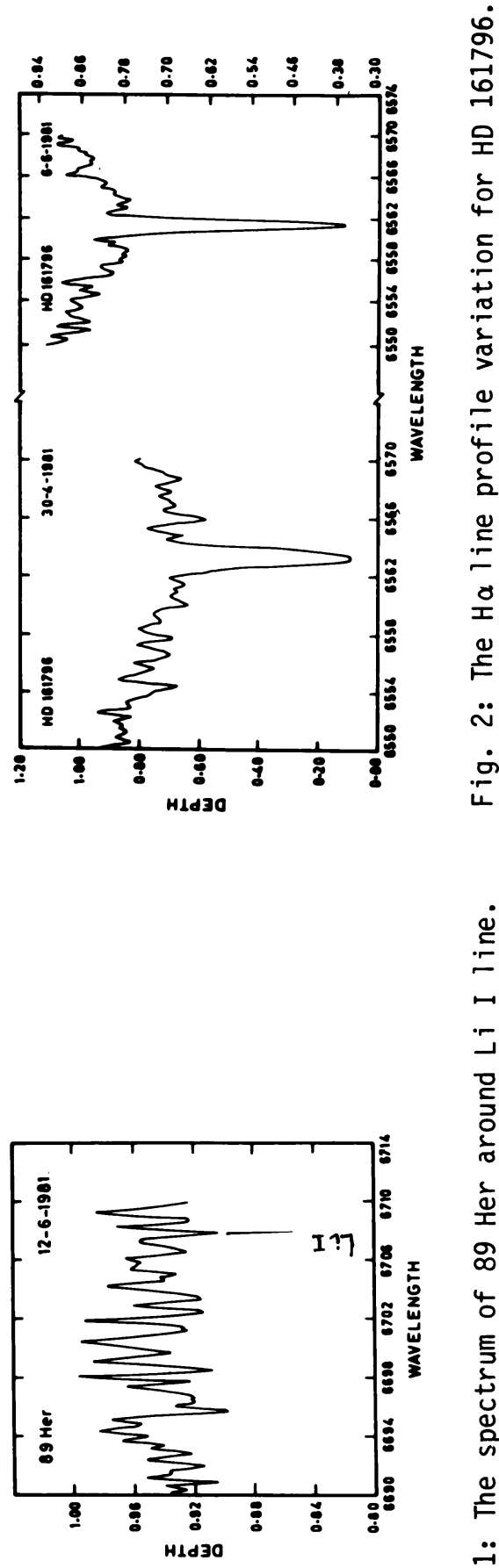

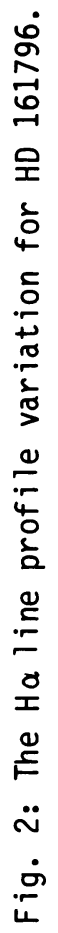

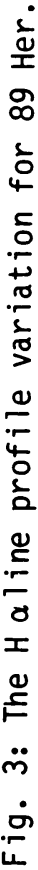


narrow absorption superposed on a broad absorption suggests a shell structure.

Our spectroscopic method of deriving atmospheric parameters is described in detail elsewhere (Girjdhar 1984). We derive $T_{\text {eff }}$ $=6000 \mathrm{~K}, \log \mathrm{g}=0.5$ and $V_{f}=4.5 \mathrm{~km} \mathrm{~s}-1$ for $89 \mathrm{Her}$ and $T_{e f f}=5600 \mathrm{~K}$, $\log g=1.5 v_{t}=4.0 \mathrm{~km} \mathrm{~s}-\mathrm{I}_{\text {for }} H D$ 161796. It was easier to measure equivalent width of LiI line in the spectrum of $89 \mathrm{Her}$ because the neighbouring lines FeI 6707.441 and VI 6708.1 are very weak in such low gravity stars. In the case of HD 161796 , due to relatively lower temperature and higher gravities the LiI line is hopelessly blended making equivalent width measurement impossible.

TABLE 1. DERIVED ABUNDANCES FOR DIFFERENT ELEMENTS

\begin{tabular}{llll}
\hline Element & No. of lines & & \\
& used & & \\
& 1 & $\mathrm{Her}$ & \\
$\mathrm{Li} / \mathrm{H}$ & 2 & 2.3 & 6.6 \\
$\mathrm{Al} / \mathrm{H}$ & 4 & 6.8 & 7.496 \\
$\mathrm{Si} / \mathrm{H}$ & 2 & $7.57 \pm 0.16$ & $7.4 \pm 0.2$ \\
$\mathrm{~S} / \mathrm{H}$ & 4 & $7.12 \pm 0.18$ & $6.1 \pm 0.25$ \\
$\mathrm{Ca} / \mathrm{H}$ & 26 & $6.2 \pm 0.18 \pm 0.16$ & $7.48 \pm 0.16$ \\
$\mathrm{Fe} / \mathrm{H}$ & & $7.57 \pm 0.15$ & \\
\hline
\end{tabular}

As is obvious from the table 1 we find almost solar abundance for $\mathrm{Si}, \mathrm{S}, \mathrm{Ca}$ and $\mathrm{Fe}$. Al shows marked overabundances in $89 \mathrm{Her}$ but a marginal one for HD 161796.

It would be of interest to derive $L i$ abundances for other members of UU Her stars and also to look for a correlation between $\mathrm{Li} / \mathrm{H}$ and ${ }^{12} \mathrm{C} /{ }^{13} \mathrm{C}$ to understand the evolutionary status of these objects.

\section{REFERENCES}

Parthasarathy,M., Pottasch,S.R. 1986, Astr. Astrophys. 154, L16. Prabhu,T.P., Anupama,G.C., Giridhar,S. 1987, Bull. Astr. Soc. India, 15,98 .

Giridhar,S. 1984, J. Astrophys. Astr. 4 , 75.

Sasselov,D. 1984, Astrophys. Space Sci. 102, 161. 
DIscussion

GRATHON The abundance of Al seems quite high. May you coment? GIRIDEAR The derived abundance is indeed quite high. But the present estimate is based on only two lines. It would be nicer including more number of lines to improve the accuracy of abundance estimates. 